\title{
Weak gratings in silicon-on-insulator for spectral filters based on volume holography
}

\author{
Marie Verbist,* Dries Van Thourhout, and Wim Bogaerts \\ Photonics Research Group, INTEC Department, Ghent University-IMEC, Center for Nano- and Biophotonics (NB-Photonics), \\ Ghent University, Sint-Pietersnieuwstraat 41, Gent 9000, Belgium \\ *Corresponding author: Marie.Verbist@UGent.be
}

Received November 26, 2012; revised January 7, 2013; accepted January 7, 2013;

posted January 8, 2013 (Doc. ID 180484); published February 1, 2013

We demonstrate integrated holographic grating filters, that can be designed toward any desired target spectrum. A straightforward design method transforms this target spectrum into a weak effective refractive index variation. The filters are implemented in high-contrast silicon-on-insulator by modulating the width of the shallow etched sides of a ridge waveguide. Measurements show that this is a promising approach to integrate flexible and versatile filters on photonic chips to enable more complex applications. (c) 2013 Optical Society of America

OCIS codes: $130.7408,050.0050,090.7330$.

Silicon-on-insulator (SOI) has emerged as a popular platform for photonic integrated circuits, mainly because of its high refractive index contrast and its compatibility with complementary metal oxide semiconductor fabrication technology [1]. As the latter technology becomes even more refined, more functionality and complexity can be added to the photonic chips. This creates a need for more flexible and versatile spectral filters. Filters based on photonic crystals, arrayed waveguide gratings, or ring resonators are well understood and have excellent performance [2,3]. Still, even with these devices it is difficult to obtain filters with complex transfer functions.

The principles of volume holography, a powerful method for encoding optical information []ㅡ, present a solution. Allowing light to travel over a large distance, through a holographic grating structure, significantly increases the opportunities for light processing. The resolution of a holographic spectral filter is closely related with the scatter strength of the grating, which means a very low refractive index contrast is desirable for highresolution filters. As SOI typically has a very high refractive index contrast, high-resolution holographic filters are not straightforward to realize in this material system. In this Letter we demonstrate holographic filters that were implemented by modulating the width of the shallow etched sides of an SOI ridge waveguide.

The filters are designed in a very direct and elegant way, translating the targeted transfer function into a weak refractive index variation. Any grating can be represented by a variation of the effective refractive index $n_{\text {eff }}$ along the propagation direction $x$ :

$$
n_{\mathrm{eff}}(x)=n_{\mathrm{eff}, 0}+\Delta n(x)
$$

A grating is weak if the index variation $\Delta n(x)$ is small $\left(\Delta n(x) \ll n_{\text {eff } 0}\right)$. Because the scatter strength is low, it is justified to neglect second-order reflections. This means that a pulse $E_{0} \delta(t)$ interacts with the grating by producing a single reflection at each point $x$ with an amplitude of $E_{R}=\alpha_{R} E_{0} \Delta n(x)$, where $\alpha_{R}$ is a constant [5]. As indicated in Fig. 1, this reflection is delayed by a transit time of $t=2 n_{\mathrm{eff}, 0} x / c$. The response to the impulse $E_{0} \delta(t)$ in reflection becomes

$$
E_{R}(t)=\alpha_{R} E_{0} \Delta n\left(\frac{c t}{2 n_{\mathrm{eff}, 0}}\right)
$$

The spectral transfer function of this filter can then be calculated as the Fourier transform of $E_{R} / E_{0}$

$$
T(f)=\mathcal{F}\left[\alpha_{R} \Delta n\left(\frac{c t}{2 n_{\mathrm{eff}, 0}}\right)\right] .
$$

Vice versa, when starting from a target transfer function, $\Delta n$ can be calculated as the inverse Fourier transform from $T(f)$.

We now want to implement the index variation $\Delta n(x)$ using a photonic waveguide, which is defined in highcontrast SOI. Many parameters affect the effective index of such an SOI waveguide: thickness, width, refractive index of core and cladding, temperature, dopants,... The grating's effective index variation $\Delta n(x)$ can thus be implemented in equally numerous ways, which will mostly depend on the available processing techniques. The difficulty is having sufficient control over the technology to define small exact variations of $n_{\text {eff }}$ with the required accuracy, because the material system has such a high inherent refractive index contrast. In this Letter, we start from a double-etched ridge waveguide, and implement $\Delta n(x)$ as a modulation of the width of the shallow etched sides, referred to as wing width $w(x)$ [6]. A schematic cross-section and top view of this waveguide grating can been seen in the gray inset of Fig. 2. The central part of the waveguides measures $220 \mathrm{~nm}$ high by $450 \mathrm{~nm}$ wide. The wing is $150 \mathrm{~nm}$ high.

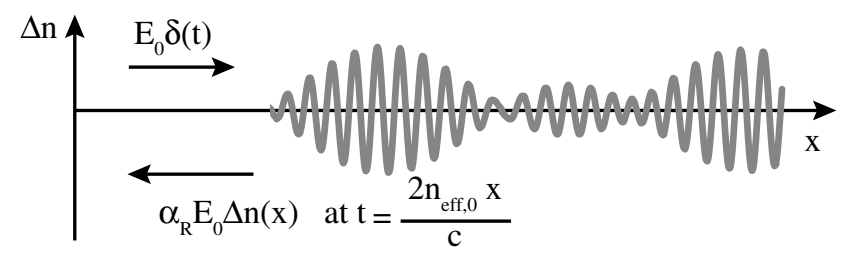

Fig. 1. Impulse response of the grating consist of one contribution from every position $x$. This contribution has a amplitude related to $\Delta n(x)$ and arrives at a time related to $x$. 
(a) $\Delta \mathbf{n}(\mathbf{x})$ from iFFT

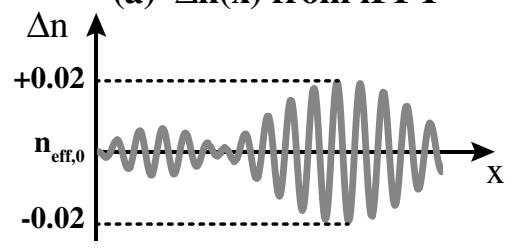

(b) $w(n)$ from simulation

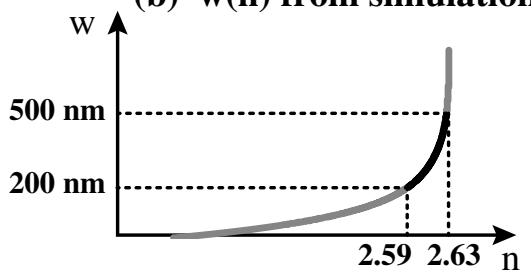

(c) ideal $\mathbf{w}(\mathbf{x})$

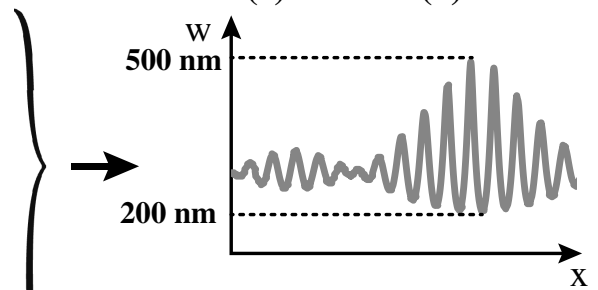

(d) $\mathbf{w}(\mathbf{x})$ for fabrication

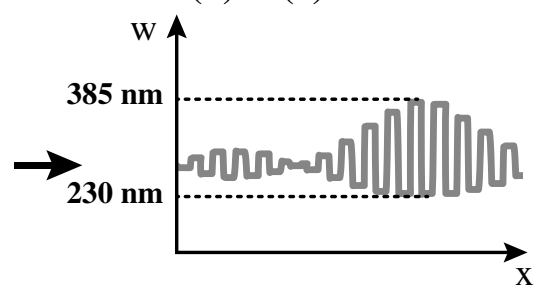

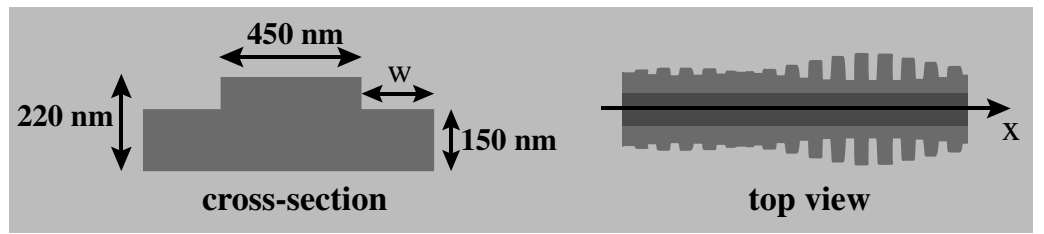

Fig. 2. Calculated (a) index variation is mapped to the (b) index dependence of the wing width. The (c) resulting ideal grating design undergoes a transformation to truncate al features that are too narrow to be fabricated. The gray inset (d) shows a schematic cross-section and top view of part of a holographic waveguide grating.

Simulations in the full-vectorial mode solver Fimmwave yield the refractive index $n$ as function of $w$, and thus the inverse: $w(n)$. By mapping the desired $\Delta n(x)$ to the index dependence $w(n)$ of the wing width, we obtain $w(x)$, the wing width as a function of the propagation length [Figs. 2(a)-2(c)]. This wing modulation causes the target spectrum to be reflected.

Depending on the fabrication scheme, additional transformations can or should be performed to satisfy fabrication requirements. For this work, the photonic chips are fabricated using $193 \mathrm{~nm}$ deep UV lithography [1]. Features narrower than $120 \mathrm{~nm}$ are not allowed by the software design rule check as they cannot be fabricated accurately. Therefore, we apply a transformation that truncates all sharp features by replacing $w(x)$ with the average value over this $120 \mathrm{~nm}$ range [Fig. 2(d)].

Using the same set of parameters, several target spectra, all consisting of a number of isolated peaks, were translated into the corresponding grating design and fabricated. As can be expected, there is a tradeoff between spectral resolution, grating length and reflected power. In principle, $\Delta n$ can be chosen arbitrarily small, resulting in an extremely high resolution. In practice, the reflections will become comparable to the scattering due to surface roughness and lose their filter strength. We set the maximal $\Delta n$ to 0.04 [Fig. 2(a)] and the grating length was chosen to be $1 \mathrm{~mm}$. For these settings, we expect a high reflected power and good resolution. $n_{\text {eff, } 0}$ can be chosen freely to ensure a practical range of the wing width. This is a range where the slope of $w(n)$ is either too shallow (very short range) or too steep (very long range). We chose a range from $200-500 \mathrm{~nm}$, which corresponds with a effective refractive index of 2.59-2.63 [Fig. 2(b)]. After truncating the narrow grating features, the wing width ranges from $230-385 \mathrm{~nm}$. When fabricated, it ranges from $250-320 \mathrm{~nm}$, due to the limitations of photolithography [7]. This corresponds to a maximal refractive index contrast of 0.012 . Figure $\underline{3}$ shows the design together with a scanning electron microscope image picture of the fabricated device. By examining a range of gratings with different contrast, this effect can be calibrated and compensated for.
The expected reflection spectrum was calculated by performing a forward Fourier transform on the final design, including all transformations. These spectra can be seen in the top row of Fig. 4. To show that the position of the peaks can be completely arbitrary, we intentionally included an irregular spectrum (middle spectrum in Fig. 4), where we omitted one of five reflection peaks.

The waveguides are equipped with grating couplers to couple light from an optical fiber to the waveguide and back. A tunable laser is used as a light source and the reflected and transmitted power are measured simultaneously using two power meters. The reflected power is measured through a circulator and index matching fluid is used between fiber and chip to reduce unwanted reflections. The polarization of the light is controlled by polarization paddles.

Figure $\underline{4}$ shows the measurements normalized with respect to the transmission of a straight waveguide with the same grating couplers. The measured spectra show distinct peaks at the same positions as the theoretical spectra. The measured reflection depends on the number of peaks in the spectrum, and is $-4.4 \mathrm{~dB}$ on average. The average peak width is $0.27 \mathrm{~nm}$ and the average side lobe suppression ratio is $-6.5 \mathrm{~dB}$. For this demonstration, no efforts were made to reduce the extent of the side lobes. This means the filter performance could still be improved dramatically.

(a) design

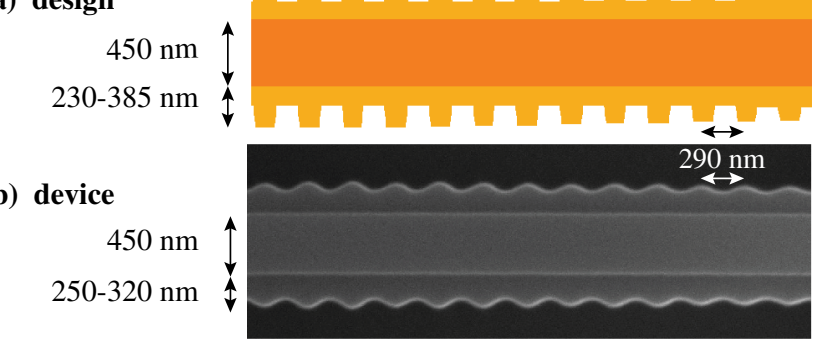

Fig. 3. (Color online) Comparing the design with the fabricated device shows that the maximal refractive index contrast of the grating is reduced. 

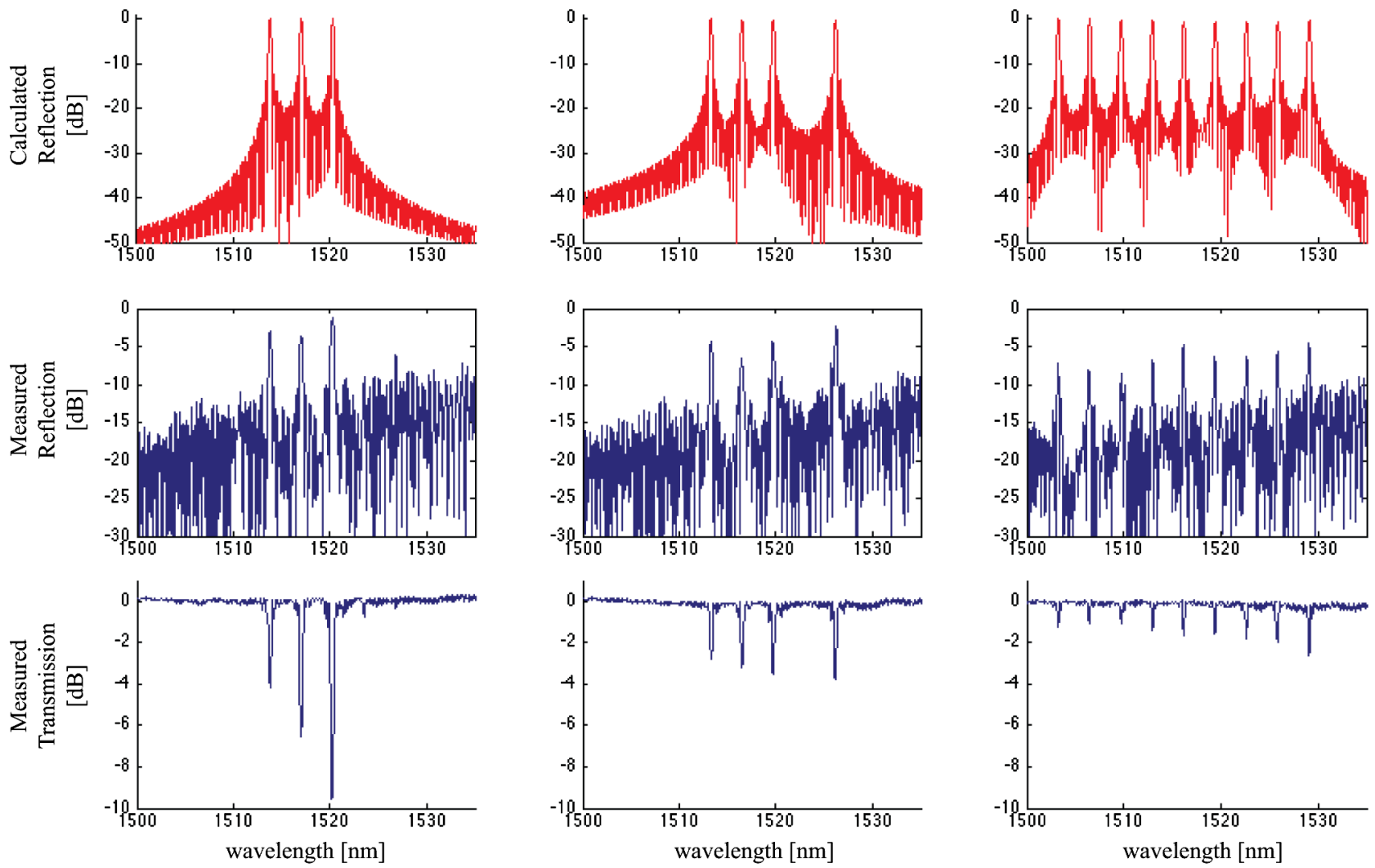

Fig. 4. (Color online) Calculated and measured spectra of three gratings with different target spectra show good correspondence.

Future work will include a calibration of the contrast reduction after fabrication, and techniques to suppress the side lobes. In addition to spectra with isolated peaks, we will also focus on more complicated customized spectra, and incorporate these in applications.

We have shown that weak holographic gratings can be implemented in an SOI waveguide to produce any targeted reflection spectrum with a tradeoff between grating length, reflected power, and spectral resolution. The design method is very straightforward as long as the index contrast is weak. The target spectrum is used as the input of the design method. It is transformed into a refractive index variation by means of an inverse Fourier transform. To obtain this weak refractive index variation in a silicon waveguide, we have used a ridge waveguide and modulated the width of the shallow etched side wings. This way, we have demonstrated devices that obtain a spectral response with several isolated peaks.

The authors acknowledge ePIXfab for the fabrication of the SOI chips. M. Verbist acknowledges the Institute for the Promotion of Innovation through Science and Technology in Flanders (IWT Vlaanderen).

\section{References}

1. S. K. Selvaraja, P. Jaenen, W. Bogaerts, D. Van Thourhout, P. Dumon, and R. Baets, J. Lightwave Technol. 27, 4076 (2009).

2. T. Tsuchizawa, K. Yamada, H. Fukuda, T. Watanabe, J. Takahashi, M. Takahashi, T. Shoji, E. Tamechika, S. Itabashi, and H. Morita, IEEE J. Sel. Topics Quantum Electron. 11, 232 (2005).

3. W. Bogaerts, S. K. Selvaraja, P. Dumon, J. Brouckaert, K. De Vos, D. Van Thourhout, and R. Baets, IEEE J. Sel. Topics Quantum Electron. 16, 33 (2010).

4. C. Peroz, A. Goltsov, S. Dhuey, P. Sasorov, B. Harteneck, I. Ivonin, S. Kopyatev, S. Cabrini, S. Babin, and V. Yankov, IEEE Photon. J. 3, 888 (2011).

5. T. Mossberg, Opt. Lett. 26, 414 (2001).

6. X. Wang, W. Shi, H. Yun, S. Grist, N. Jaeger, and L. Chrostowski, Opt. Express 20, 15547 (2012).

7. W. Bogaerts, P. Bradt, L. Vanholme, P. Bienstman, and R. Baets, Opt. Quantum Electron. 40, 801 (2008). 